\title{
A Hybrid TDOA/RSSD Geolocation System using the Unscented Kalman Filter
}

\author{
Noha El Gemayel, Holger Jäkel and Friedrich K. Jondral \\ Communications Engineering Lab, Karlsruhe Institute of Technology (KIT), Germany \\ \{noha.gemayel, holger.jaekel, friedrich.jondral\}@kit.edu
}

\begin{abstract}
Geolocation is an important topic nowadays. Among other applications, geolocation can be used by frequency regulators to monitor the time, frequency and space domain. In this case of a passive geolocation system, Time Difference of Arrival (TDOA) is often used. In this paper we analyze a geolocation system based on a combination of TDOA and Received Signal Strength Differences (RSSD). The benefit of combining the two measurement types is shown using the Cramer Rao Lower Bound (CRLB). The suitability of the Unscented Kalman Filter (UKF) as an algorithm for the hybrid geolocation system is investigated. Simulation results show that by using the UKF, the instability of RSSD geolocation, that is often caused by bad geometry, is compensated. The UKF nearly approaches the CRLB and greatly outperforms the previously proposed least squares hybrid solution. Using the UKF, the information about the signal power can be definitely used to enhance the position estimation accuracy.
\end{abstract}

\section{INTRODUCTION}

Time Difference of Arrival (TDOA) offers a good solution for passive geolocation systems that are often deployed for security, emergency as well as regulatory reasons. TDOA provides a reasonable accuracy in many scenarios and only requires precise clocks. In the literature, many papers have dealt with solving the hyperbolic equations of TDOA using closed form or iterative algorithms [1], [2], [3]. Kalman Filters offer another solution method due to their high robustness against errors. Since the system of TDOA equations is nonlinear, a special type of Kalman Filters can be used. While the Extended Kalman Filter [4] linearizes the equations around an estimate, the Unscented Kalman Filter (UKF) [5],[6] uses the whole dynamics of the system without linearization on the so called Sigma Points.

Among other error sources, TDOA suffers from low accuracy because of bad synchronization among the sensors or because of narrow bandwidths of the signals to be localized. In that case, other measurements can be used to enhance the location estimation accuracy. For a low cost passive sensor network, Received Signal Strength Differences (RSSD) can add information to the localization problem. Some algorithms were introduced to solve the localization equation using the RSSD measurements [7]. In [8] and [9], the least squares solution to the hybrid system using both RSSD and TDOA measurements is presented. The proposed method is based on linearizing the system equations around an estimate and solving the least squares solution. Unfortunately, the method suffers from large errors in many scenarios.
This paper investigates the case of enhancing the position estimate accuracy of a TDOA system using RSSD measurements. The paper proposes the Unscented Klaman Filter as a novel hybrid geolocation algorithm using both the signal attenuation information and the TDOA measurements. The paper is organized as follows. Section II presents the system model of TDOA and RSSD. The benefits of using a hybrid estimation system with both measurement types are shown by the Cramer Rao Lower Bound (CRLB). Section III presents the equations of the Unscented Kalman Filter using the hybrid measurements. Section IV shows simulation results and compares them to the previously proposed hybrid method. Section $\mathrm{V}$ concludes the paper and suggests ideas for further work.

\section{TDOA AND RSSD}

In this section we analyze position estimation systems that use TDOA, RSSD or both measurements combined. The geometry behind the measurements as well as the best possible accuracy of the system given by the CRLB are presented. The localization system described in this paper is based on $N$ sensors in a two dimensional plane with known coordinates $\left[x_{i}, y_{i}\right], \quad i=1, \ldots, N$ which can record time stamped IQ data in a certain bandwidth. The signal of interest is sent from a transmitter at the unknown position $[x, y]$.

\section{A. Time Difference of Arrival}

The received signals at two different sensors are crosscorrelated to obtain the time difference of arrival estimate $\hat{\tau}_{i, j}$. Measurement errors due to synchronization errors and channel effects are modelled as added white gaussian noise. The estimated distance difference between the transmitter and sensors $i$ and $j$ is modelled by:

$$
\begin{aligned}
& \begin{aligned}
\hat{d}_{i, j}= & d_{i, j}+\eta_{j}-\eta_{i} \\
& =\hat{\tau}_{i, j} \cdot c, \quad i, j=1, \ldots, N, \quad i \neq j
\end{aligned} \\
& \text { with } \quad \eta_{i} \in \mathcal{N}\left(0, \sigma_{T_{i}}^{2}\right) \\
& \begin{aligned}
d_{i, j}= & d_{j}-d_{i} \\
d_{i}= & \sqrt{\left(x_{i}-x\right)^{2}+\left(y_{i}-y\right)^{2}}
\end{aligned}
\end{aligned}
$$

where $c$ is the propagation speed and $d_{i}$ is the Euclidean distance between the transmitter and sensor $i$. The terms $\eta_{i}, \eta_{j}$ describe the additive white noise. Solving equation (1) for $[x, y]$ resolves in the hyperbolic TDOA equation system. It is usually referenced to one sensor, e.g., sensor one. Assuming 
an unbiased estimate, the Cramer Rao Lower Bound (CRLB) of the position estimate using TDOA measurements can be calculated using the Fisher information matrix [1]:

$$
\mathbf{F}_{T}=\mathbf{G}^{T} \mathbf{Q}_{T}^{-1} \mathbf{G}
$$

with

$$
\mathbf{G}=\left(\begin{array}{cc}
\frac{x_{1}-x}{d_{1}}-\frac{x_{2}-x}{d_{2}} & \frac{y_{1}-y}{d_{1}}-\frac{y_{2}-y}{d_{2}} \\
\frac{x_{1}-x}{d_{1}}-\frac{x_{3}-x}{d_{3}} & \frac{y_{1}-y}{d_{1}}-\frac{y_{3}-y}{d_{3}} \\
\ldots & \ldots \\
\frac{x_{1}-x}{d_{1}}-\frac{x_{N}-x}{d_{N}} & \frac{y_{1}-y}{d_{1}}-\frac{y_{N}-y}{d_{N}}
\end{array}\right)
$$

and $\mathbf{Q}_{T}$ being the covariance matrix of the distance difference estimates $\hat{d}_{i, j}$. Estimators based on TDOA measurements use the distance differences $d_{i, j}$ rather than the time differences. They either linearize the hyperbolic equations around an estimate to calculate a least squares solution [2] or they produce a closed form solution [1]. Extended and Unscented Kalman Filters also form possible estimators for that problem either by linearizing the nonlinear equations or by approximating the system probability distribution.

\section{B. Received Signal Strength Difference}

In this method, the received signal power at sensor $i$ is modelled as:

$$
\hat{P}_{R i}=P_{T} \cdot d_{i}^{-\gamma} \cdot l_{i}
$$

where $P_{T}$ is the transmitted signal power, $\gamma$ is a path loss exponent and is assumed to be known to the receivers, and $d_{i}$ is the distance given in equation (1). $l_{i}$ denotes log normal fading, i.e.,

$$
l_{i}=10^{n_{i}} \quad n_{i} \in \mathcal{N}\left(0, \sigma_{P_{i}}^{2}\right) .
$$

Expressing equation (4) in $\mathrm{dB}$ and substracting the power measurements of two sensors, we obtain the relation between the received signal strength differences and the transmitter position.

$$
\begin{aligned}
\hat{\Omega}_{i} & =10 \cdot \log _{10}\left(\hat{P}_{R i}\right)=C-10 \cdot \gamma \cdot \log _{10}\left(d_{i}\right)+n_{i} \\
\hat{\Omega}_{i, k} & =\hat{\Omega}_{i}-\hat{\Omega}_{k} \\
& =10 \cdot \gamma \cdot \log _{10}\left(d_{k}\right)-10 \cdot \gamma \cdot \log _{10}\left(d_{i}\right)+n_{i}-n_{k}
\end{aligned}
$$

The Fisher information matrix of an unbiased estimator for RSSD measurements was presented in [10]. Using

$$
\begin{aligned}
w_{k} & =\left[\sum_{i=1}^{N} \sigma_{P_{i}}^{-2}\right]^{-1} \cdot \sigma_{P_{k}}^{-2} \\
a_{k} & =\frac{x-x_{k}}{d_{k}^{2}}, \quad b_{k}=\frac{y-y_{k}}{d_{k}^{2}} \\
\chi & =\sum_{k=1}^{N} w_{k} a_{k} \quad \nu=\sum_{k=1}^{N} w_{k} b_{k} \\
\xi & =\sum_{k=1}^{N} w_{k} a_{k}^{2} \quad \psi=\sum_{k=1}^{N} w_{k} b_{k}^{2} \\
\rho & =\sum_{k=1}^{N} w_{k} a_{k} b_{k}
\end{aligned}
$$

the Fisher information matrix is given by:

$$
\mathbf{F}_{P}=\left(\sum_{k=1}^{N} \sigma_{P_{k}}^{-2}\right) \cdot\left(\frac{10 \gamma}{\ln (10)}\right)^{2} \cdot\left(\begin{array}{cc}
\xi-\chi^{2} & \rho-\chi \cdot \nu \\
\rho-\chi \cdot \nu & \psi-\nu^{2}
\end{array}\right)
$$

Compared to the TDOA geometry based on hyerbolas, solving the RSSD equations resolves in distance ratios $\frac{d_{i}}{d_{j}}$ resembling circles with possible transmitter positions. The geometry of the system is far more complex than that of the TDOA system. Inaccuracies due to linearization around an estimate cause much larger errors. Estimators for RSSD geolocation systems were presented in [10] and [7].

\section{Hybrid TDOA and RSSD}

In this paper, we analyze the performance of a TDOA geolocation system, which can benefit from the extra information given by the RSSDs. Assuming that the noise terms of the two measurement types are independent, the joint probability density function can be expressed as the product of the two probability density functions. The Fisher information matrix of the hybrid system is the sum of the two matrices. In this case,

$$
\mathbf{F}_{\text {Hybrid }}=\mathbf{F}_{T}+\mathbf{F}_{P} .
$$

The bound of the minimum mean Euclidean distance between the estimate and the true position is determined using the trace of the inverse matrix. Fig. 1 and Fig. 2 show an example of the Cramer Rao Lower Bound of the hybrid system over $\sigma_{T}$ and $\sigma_{P}$ assuming equal standard deviations for all sensors. The results can be compared to the case where only TDOA or RSSD measurements are used. While the TDOA error increases linearly with growing standard deviation, the curve of the hybrid system grows smoother, the better the RSSD measurements are. The bound can only get lower with the additional information. Whenever the TDOA measurements are too erroneous, for example because of a low signal bandwidth, calculating the received signal strength differences can greatly enhance the result. Furthermore, Fig. 2 shows the instability of the RSSD geolocation system. Estimating the transmitter position with the RSSD measurements only is 


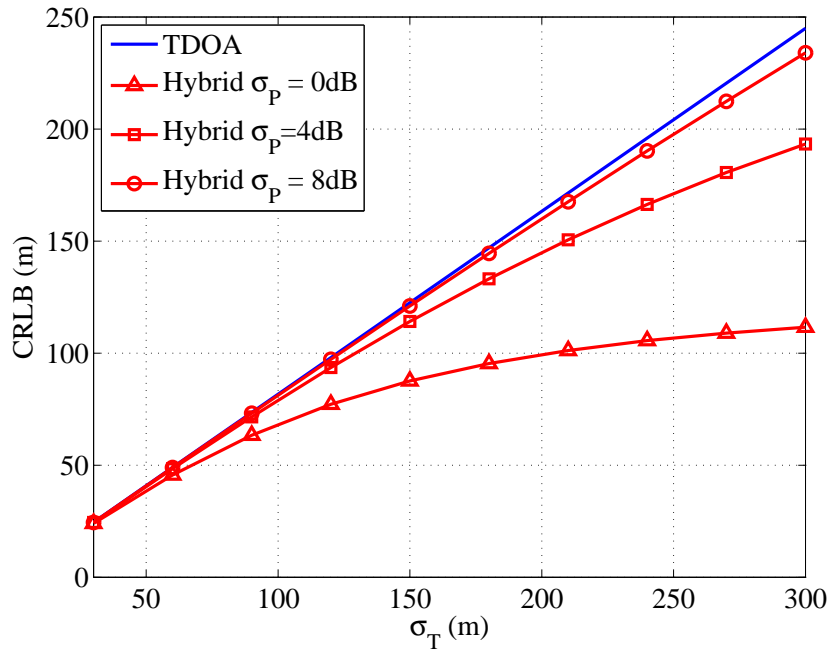

Fig. 1. CRLB of the conventional TDOA approach compared to the Hybrid approach

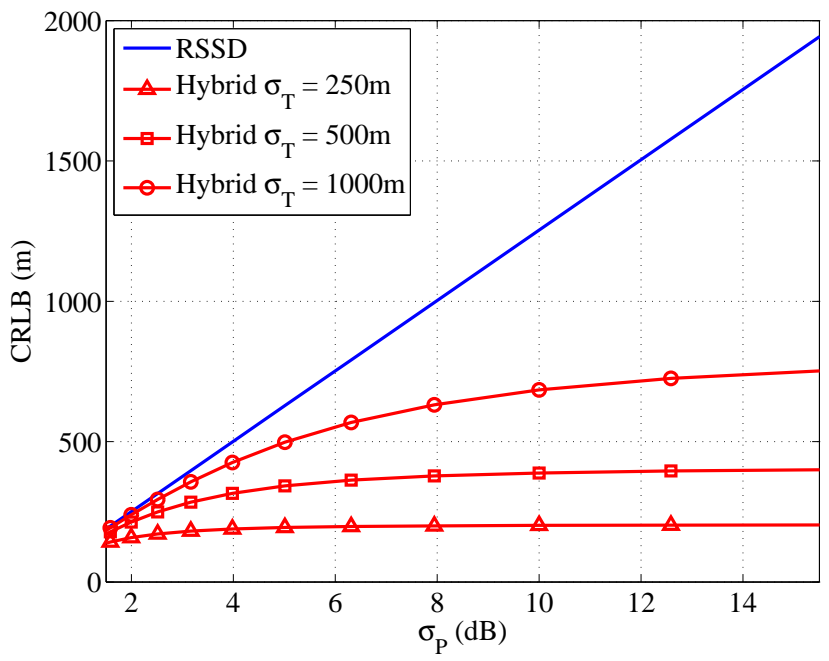

Fig. 2. CRLB of the conventional RSSD approach compared to the Hybrid approach

too inaccuracte and instable. Gathering both types of measurements can contribute to a better estimation, especially in cases where TDOA measurements are not sufficiently accurate. Next, it is important to integrate the RSSD measurements in a way that doesn't decrease system accuracy.

\section{The Unscented FILTER EQUATIONS Using TDOA AND RSSD MEASUREMENTS}

The Unscented Filter is a Kalman Filter for nonlinear estimation. The filter approximates the probablity distribution of the system at each time step $k$ [11]. The system equations of the Kalman Filter are generally given as:

$$
\begin{aligned}
\mathbf{x}(k+1) & =\mathbf{f}(\mathbf{x}(k))+\mathbf{u}(k) \\
\mathbf{z}(k) & =\mathbf{h}(\mathbf{x}(k))+\mathbf{v}(k)
\end{aligned}
$$

where $\mathbf{x}$ is the state vector, $\mathbf{f}$ is the state function and $\mathbf{u}$ is the added system noise and where $\mathbf{z}$ is the measurement, $\mathbf{h}$ the measurement function and $\mathbf{v}$ is the measurement noise. For the sake of simplicity, we consider here stationary transmitters, so that the state doesn't change from one step to another and the state function can be described as unity matrix.

The filter uses the Unscented Transformation on each state. In this transformation, a cloud of points, the Sigma Points, undergo the dynamics of the nonlinear system with the aim of calculating the statistics of the random variable. The generated Sigma Points should preserve the mean and the covariance of the system and are therefor chosen deterministically. Many methods were proposed for generating the Sigma Points. Here, the method described in [6]. was used. The steps of the Kalman Filter are presented next.

\section{A. Initializing}

The algorithm starts with an initial state vector consisting of an estimate of the transmitter position and an initial estimate of the state covariance:

$$
\begin{aligned}
\hat{\mathbf{x}}(0) & =E[\mathbf{x}(0)] \\
\hat{\mathbf{P}}(0) & =E\left[(\mathbf{x}-\hat{\mathbf{x}}(0))(\mathbf{x}-\hat{\mathbf{x}}(0))^{T}\right]
\end{aligned}
$$

\section{B. Generating Sigma Points}

The next step consists of updating the state and covariance according to the equation (15) and calculating the predicted mean and covariance of the state using Sigma Points. Due to the fact that we are assuming a stationary transmitter, the predicted mean and covariance of the state are equal to the state vector and the covariance matrix of the previous step.

$$
\begin{aligned}
\mathbf{x}^{*}(k+1) & =\hat{\mathbf{x}}(k) \\
\mathbf{P}^{*}(k+1) & =\hat{\mathbf{P}}(k)
\end{aligned}
$$

where $\left(^{*}\right)$ stands for predicted parameters. At each step, the Sigma Points $\mathbf{x}_{0}, \mathbf{x}_{1} \ldots \mathbf{x}_{2} N_{\text {dim }}+1$ are generated around the predicted state with the state dimension $N_{d i m}$ using the predicted covariance.

$$
\begin{aligned}
\mathbf{x}_{0} & =\mathbf{x}^{*}(k+1) \\
\mathbf{x}_{i} & =\mathbf{x}_{0}+\left(\sqrt{\frac{N_{d i m}}{1-W_{0}} \mathbf{P}^{*}(k+1)}\right)_{i} \\
\mathbf{x}_{i+N_{d i m}} & =\mathbf{x}_{0}-\left(\sqrt{\frac{N_{d i m}}{1-W_{0}} \mathbf{P}^{*}(k+1)}\right)_{i} \\
W_{i} & =\frac{1-W_{0}}{2 N_{d i m}} \quad i=1,2, \ldots N_{d i m}
\end{aligned}
$$

where ()$_{i}$ denotes the $i$ th row of the matrix square root of a positive semidefinite matrix. $W_{0}, W_{i}$ are the according weights of the points and $W_{0}$ is a tuning parameter than can take any value except for 1 . 


\section{Predicting Measurements}

The Sigma Points are projected through the measurement function $\mathbf{h}$, which means that each Sigma Point is regarded as a transmitter position and the according TDOA and RSSD measurements are calculated using the measurement equations (1) and (6). The resulting measurement vector for Sigma Point $i$ has the following form:

$\mathbf{z}_{i}=\mathbf{h}\left(\mathbf{x}_{i}\right)=\left[\hat{d}_{2,1}, \hat{d}_{3,1}, \ldots . \hat{d}_{N-1,1}, \hat{\Omega}_{2,1}, \hat{\Omega}_{3,1}, \ldots ., \hat{\Omega}_{N-1,1}\right]^{T}$

The predicted measurement is the weighted sum of the projected Sigma Points given as:

$$
\mathbf{z}^{*}(k+1)=\sum_{i=0}^{2 N_{\text {dim }}} W_{i} \mathbf{z}_{i}
$$

\section{Calculating Innovation and Cross Covariance Matrices}

The predicted measurement covariance matrix and the cross covariance matrix can be calculated by:

$$
\begin{aligned}
& \mathbf{P}_{z z}=\sum_{i=0}^{2 N_{\text {dim }}} W_{i}\left[\mathbf{z}_{i}-\mathbf{z}^{*}(k+1)\right]\left[\mathbf{z}_{i}-\mathbf{z}^{*}(k+1)\right]^{T}+\mathbf{Q}_{H y b} \\
& \mathbf{P}_{x z}=\sum_{i=0}^{2 N_{\text {dim }}} W_{i}\left[\mathbf{x}_{i}-\mathbf{x}^{*}(k+1)\right]\left[\mathbf{z}_{i}-\mathbf{z}^{*}(k+1)\right]^{T}
\end{aligned}
$$

$\mathbf{Q}_{H y b}$ is the measurement covariance matrix that has the following form:

$$
\mathbf{Q}_{H y b}=\left(\begin{array}{cc}
\mathbf{Q}_{T} & \mathbf{0} \\
\mathbf{0} & \mathbf{Q}_{P}
\end{array}\right)
$$

where $\mathbf{Q}_{\mathbf{T}}$ is the covariance matrix of the TDOA measurements and $\mathbf{Q}_{\mathbf{P}}$ is the covariance matrix of the RSSD measurements.

\section{E. Correcting and Updating}

The filtering step consists of calculating the Kalman Gain $\mathbf{K}$ and performing the measurement update equations with $\mathbf{z}(k+$ $1)$ as the observed measurement vector with the same elements as in equation (19).

$$
\begin{aligned}
\mathbf{K}(k+1) & =\mathbf{P}_{x z} \mathbf{P}_{z z}^{-1} \\
\hat{\mathbf{x}}(k+1) & =\mathbf{x}^{*}(k+1)+\mathbf{K}(k+1)\left[\mathbf{z}^{*}(k+1)-\mathbf{z}(k+1)\right] \\
\hat{\mathbf{P}}(k+1) & =\mathbf{P}^{*}(k+1)+\mathbf{K}(k+1) \mathbf{P}_{z z} \mathbf{K}^{T}(k+1)
\end{aligned}
$$

\section{Simulation AND Results}

In this section we present the simulated scenarios and some important results. For the geometry of the system, 6 Sensors were symmetrically distributed around an origin point (here: [300 300]) with a radius of $2000 \mathrm{~m}$ and the transmitter was put in 10 different positions with the goal of covering both good and bad geometry conditions. The simulated positions can be seen in Fig. 3.

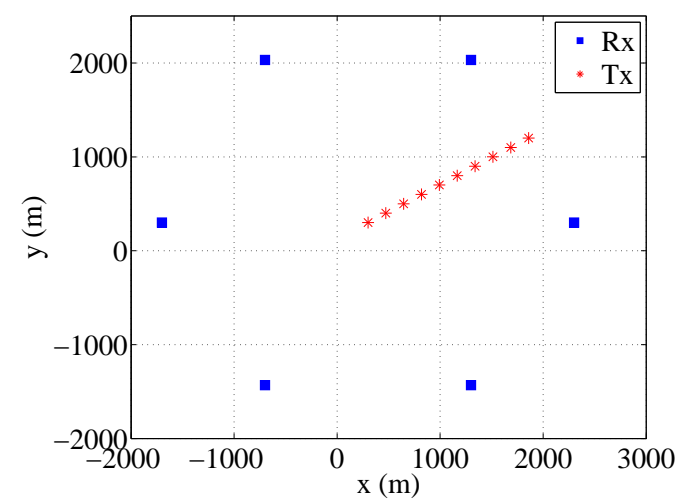

Fig. 3. Simulated transmitter and sensor positions

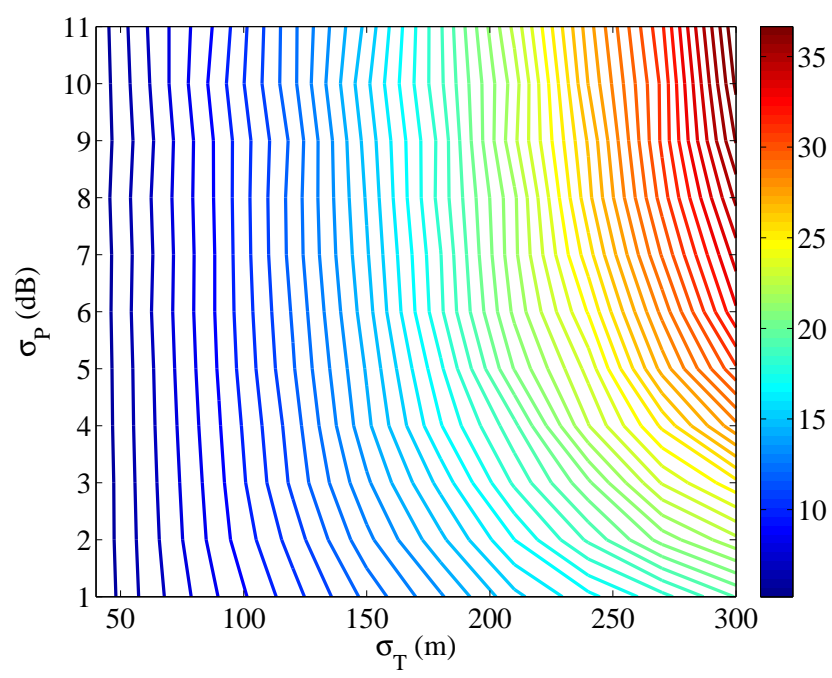

Fig. 4. Contour graph of the RMSE over varying standard deviations

Fig. 4 shows the contour graph of the root mean square error (RMSE) of the position estimate after 100 Kalman filter steps. The results are plotted over growing standard deviations of TDOA and RSSD measurements. It can be seen that with decreasing TDOA accuracy, the additional RSSD measurements are helpful. The more accurate the RSSDs are, the better the result of the hybrid system compared to the TDOA system. The figure also shows how the RMSE is relatively insensitive to the RSSD accuracy. It is important to mention here that the simulated geometry is not the best case for RSSD measurements and that other scenarios can benefit more from the hybrid system.

Fig. 5 compares the results of using the UKF as a hybrid geolocation algorithm to the results of using the linearized least squares algorithm presented in [8]. The standard deviation of the TDOAs was set to $150 \mathrm{~m}$. The plot shows the RMSE after 300 simulation steps over growing RSSD measurement standard deviation. To be able to compare both methods fairly, the RSSD measurements were averaged in each step before undergoing the least squares algorithm. As a reference, both the CRLB of the position estimate using 


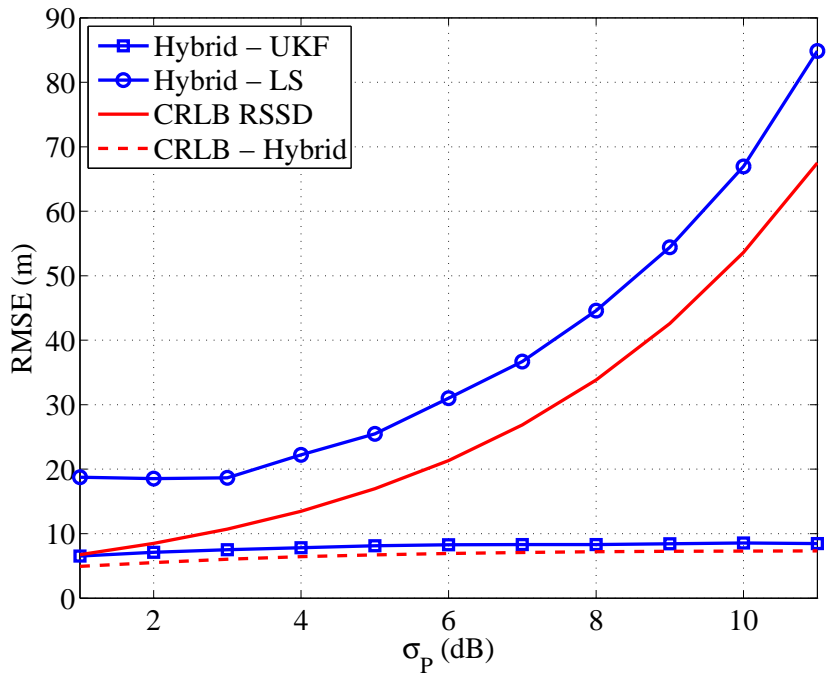

Fig. 5. RMSE of the linearized least squares hybrid method compared to the proposed hybrid method

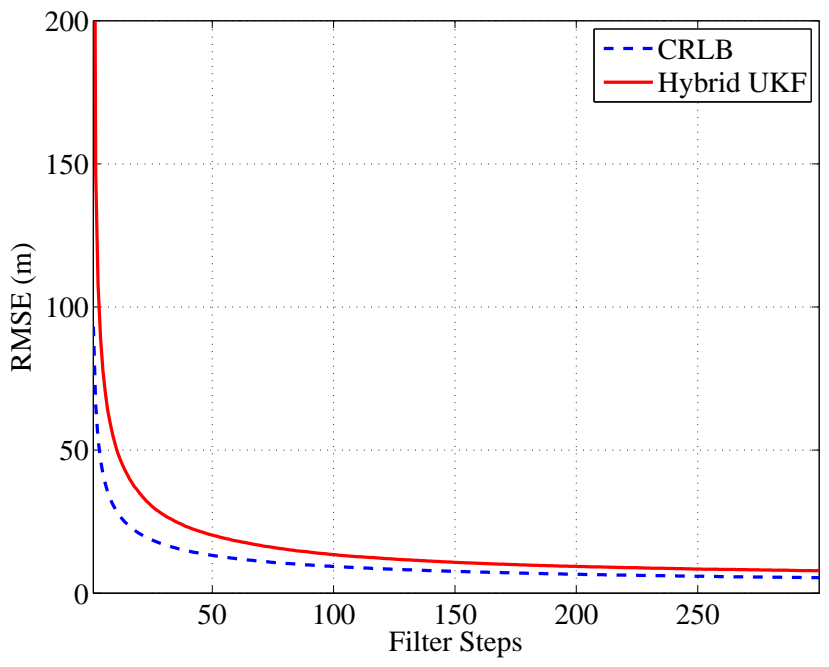

Fig. 6. Position estimates using the UKF compared to the CRLB

RSSD and that using hybrid measurements are plotted. The results of the least squares algorithm are greatly influenced by the RSSD measurements and therefor grow accordingly parallel to the CRLB, the UKF results are much smoother and nearly approach the CRLB of the hybrid system, which means that the UKF can benefit from the additional information without adding error or inaccuracy due to linearizing the RSSD equations.

Fig. 6 shows the results of the Unscented Kalman Filter over
300 filter steps. This plot shows how the filter converges to the Cramer Rao Bound with each step. This proves again how the UKF can optimally make use of the available additional information given by the RSSDs.

\section{SUMMARY AND FUTURE WORK}

In this paper we presented a hybrid geolocation method using Time Difference of Arrival and Received Signal Strength Difference measurements. The idea behind the method is to benefit from the information of the signal power in a passive TDOA system while preventing additional accuracy loss. For that reason, the Unscented Kalman Filter was presented as a more reliable approach compared to the least squares hybrid solution. The Kalman Filter approaches the Cramer Rao Lower Bound of the hybrid system with each filter step. In future work, we analyze the effect of unknown path loss exponents and we investigate the idea of estimating the exponent as a parameter in the Kalman Filter.

\section{REFERENCES}

[1] Y. Chan and K. Ho, "A simple and efficient estimator for hyperbolic location," IEEE Transactions on Signal Processing, vol. 42, no. 8, pp. $1905-1915$, aug 1994.

[2] W. Foy, "Position-location solutions by taylor-series estimation," IEEE Transactions on Aerospace and Electronic Systems, vol. AES-12, no. 2, pp. $187-194$, march 1976.

[3] B. Friedlander, "A passive localization algorithm and its accuracy analysis," IEEE Journal of Oceanic Engineering, vol. 12, no. 1, pp. $234-245$, january 1987.

[4] C. Hongyang, D. Ping, X. Yongjun, and L. Xiaowei, "A robust location algorithm with biased extended kalman filtering of tdoa data for wireless sensor networks," in Proceedings of the 2005 International Conference on Wireless Communications, Networking and Mobile Computing, vol. 2, sept. 2005 , pp. $883-886$.

[5] C. Savage, R. Cramer, and H. Schmitt, "Tdoa geolocation with the unscented kalman filter," in International Conference on Networking, Sensing and Control, 2006. ICNSC '06. Proceedings of the 2006 IEEE, 0-0 2006, pp. $602-606$.

[6] S. Julier and J. Uhlmann, "Unscented filtering and nonlinear estimation," Proceedings of the IEEE, vol. 92, no. 3, pp. 401 - 422, mar 2004.

[7] S. Wang and R. Inkol, "A near-optimal least squares solution to received signal strength difference based geolocation," in IEEE International Conference on Acoustics, Speech and Signal Processing (ICASSP), 2011, may 2011, pp. $2600-2603$.

[8] R.-T. Juang, D.-B. Lin, and H.-P. Lin, "Hybrid sadoa/tdoa mobile positioning for cellular networks," Communications, IET, vol. 1, no. 2, pp. $282-287$, april 2007.

[9] - "Hybrid sadoa/tdoa location estimation scheme for wireless communication systems," in Vehicular Technology Conference, 2006. VTC 2006-Spring. IEEE 63rd, vol. 3, may 2006, pp. $1053-1057$.

[10] S. Wang, R. Inkol, and B. Jackson, "Relationship between the maximum likelihood emitter location estimators based on received signal strength (rss) and received signal strength difference (rssd)," in 26th Biennial Symposium on Communications (QBSC), 2012, may 2012, pp. 64 -69.

[11] E. Wan and R. van der Merwe, "The unscented kalman filter for nonlinear estimation," in Adaptive Systems for Signal Processing, Communications, and Control Symposium 2000. AS-SPCC. The IEEE 2000, 2000, pp. $153-158$. 